\title{
The prognostic value and non-invasive predictors of splenomegaly in cirrhotic patients with hepatocellular carcinoma following curative resection
}

\author{
Li Zhou ${ }^{A-F}$, Shao-Bin Wang ${ }^{B}$, Shu-Guang Chen ${ }^{B}$, Qiang Qu ${ }^{C}$, Jing-An Rui ${ }^{(-E}$ \\ Department of General Surgery, Peking Union Medical College Hospital, Beijing, China \\ A - research concept and design; $\mathrm{B}$ - collection and/or assembly of data; $\mathrm{C}$ - data analysis and interpretation; \\ $D$ - writing the article; $E$ - critical revision of the article; $F$ - final approval of the article
}

Address for correspondence

Li Zhou

E-mail:lizhou02@hotmail.com

Funding sources

None declared

Conflict of interest

None declared

Received on September 8, 2017

Reviewed on December 7, 2017

Accepted on January 6, 2018

Published online on August 3, 2020

Cite as

Zhou L, Wang S-B, Chen S-G, Qu Q, Rui J-A. The prognostic value and non-invasive predictors of splenomegaly in cirrhotic patients with hepatocellular carcinoma following curative resection. Adv Clin Exp Med. 2020;29(7):879-886. doi:10.17219/acem/81935

DOI

10.17219/acem/81935

Copyright

Copyright by Author(s)

This is an article distributed under the terms of the

Creative Commons Attribution Non-Commercial License

(http://creativecommons.org/licenses/by-nc-nd/4.0/)

\begin{abstract}
Background. The prognostic value and non-invasive predictors of splenomegaly in cirrhotic patients with hepatocellular carcinoma (HCC) after curative resection remain unknown.

Objectives. To investigate the prognostic value and non-invasive predictors of splenomegaly in cirrhotic patients with HCC after curative resection.

Material and methods. The medical records of 78 patients with HCC and liver cirrhosis who underwent curative resection were retrospectively reviewed. The influence of spleen size, measured with clinically routine ultrasonography (USG), on overall and disease-free survival was evaluated using univariate and multivariate analyses. The efficiency of some frequently used blood-derived liver function parameters and non-invasive fibrosis markers to predict splenomegaly was also assessed.

Results. It was shown that tumor size $>5 \mathrm{~cm}$, the presence of microvascular invasion, tumor-node metastasis (TNM) stage III-IVA of the tumor, spleen size $>11.45 \mathrm{~cm}$, and age $\leq 52$ years were associated with poor overall survival and/or disease-free survival in univariate analyses (all $p<0.05$ ). In multivariate analyses, spleen size was identified as an independent predictor for both overall and disease-free survival $(p<0.001$ and $p=0.012$, respectively). On the other hand, platelet count, aspartate aminotransferase (AST) to platelet ratio index (APRI) and Fibrosis-4 index (FIB-4) scores were significantly different between small and large spleen groups ( $p=0.026,0.003$ and 0.003 , respectively), while statistical differences for albumin, alanine aminotransferase (ALT), AST, total bilirubin, AST to ALT ratio (AAR), and age-platelet index (API) were not found. Using receiver operating characteristic (ROC) curves, high powers of platelet count, APRI and FIB-4 in splenomegaly prediction were confirmed.
\end{abstract}

Conclusions. Splenomegaly, which can be predicted by some non-invasive variables, serves as a strong determinant for postresectional prognoses of cirrhotic patients with HCC.

Key words: splenomegaly, prognosis, hepatocellular carcinoma, non-invasive markers 


\section{Introduction}

Hepatocellular carcinoma (HCC) is well-known as a malignant neoplasm with high incidence and mortality worldwide. ${ }^{1}$ According to recent epidemiological data from China, population growth and ageing led to large and rising number of new cases of HCC in 2015, despite its declining rate. ${ }^{2}$ In addition, the overall long-term prognosis of HCC remains disappointing, although surgical therapies, including curative resection and liver transplantation, produce the best results in highly selected patients. ${ }^{1}$ Therefore, factors associated with the outcome of HCC have long been of interest. Previous studies have identified many clinical and pathologic variables as prognosticators of HCC patients who had undergone hepatectomies. ${ }^{3-7}$ The majority of them were tumor-related, such as microvascular invasion (MVI), serum $\alpha$-fetoprotein (AFP) level, histological grade, tumor-node-metastasis (TNM) stage, portal vein tumor thrombus, as well as tumor size and number. ${ }^{3-7}$ However, it is important to note that some authors found that liver cirrhosis correlated with postresectional survival of $\mathrm{HCC}$ patients, indicating that the prognosis of $\mathrm{HCC}$ was determined not only by the tumor itself, but also by the background liver status. 3,6

Splenomegaly is one of the common clinical signs of portal hypertension that is often ascribed to liver cirrhosis. Although portal hypertension has been shown to be prognostic in patients following hepatectomy, counterexamples have also been reported. ${ }^{6,8-13}$ In addition, whether splenomegaly influences the long-term prognosis of patients with resected HCC remains unclear. The present study was designed to elucidate the impact of splenomegaly on the long-term prognosis of cirrhotic patients with HCC who had undergone curative resections. The efficiency of some frequently used blood-derived liver function parameters and non-invasive fibrosis markers to predict splenomegaly in this subgroup of HCC was also assessed.

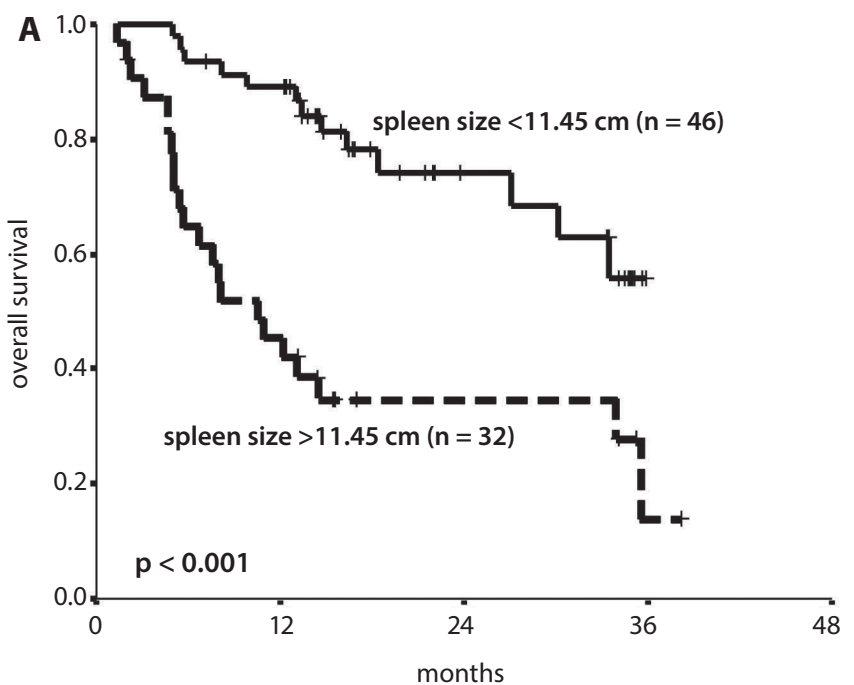

\section{Material and methods}

\section{Patients and variables}

The study involved 78 patients with HCC and liver cirrhosis who had undergone curative resection. Twelve factors, including the patient's general situation (age and gender) and liver background status (hepatitis B surface antigen (HBsAg), spleen size and clinically significant portal hypertension (CSPH)) as well as tumor-related factors (serum AFP value, capsule completeness, MVI, TNM stage, histological grade, as well as tumor size and number) were included in survival analyses. Clinically significant portal hypertension was diagnosed using the standard surrogate criteria, i.e., the presence of gastroesophageal varices or splenomegaly $>12 \mathrm{~cm}$ with a platelet count $<100 / \mathrm{mm}^{2} .{ }^{10}$ Tumor-node-metastasis stages were based on the $7^{\text {th }}$ edition of the American Joint Committee on Cancer (AJCC) Staging Manual for HCC. ${ }^{14}$ The Edmondson-Steiner

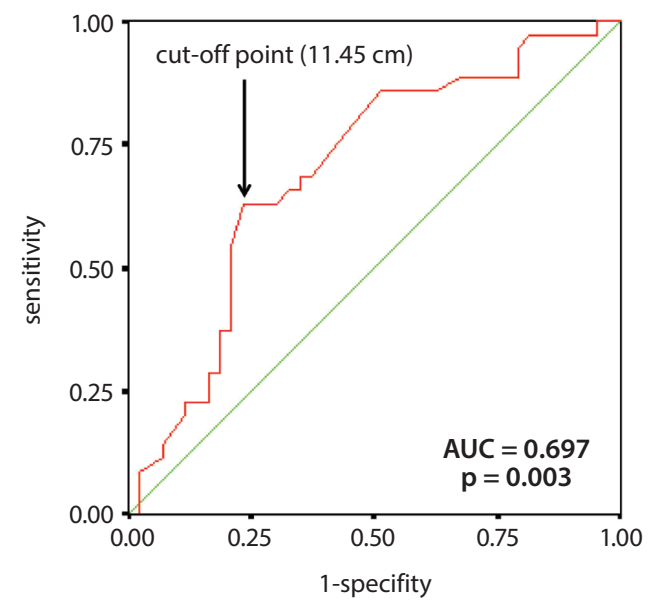

Fig. 1. Determination of the cut-off value of spleen size for overall survival based on the receiver operating characteristic (ROC) curve; AUC - area under curve

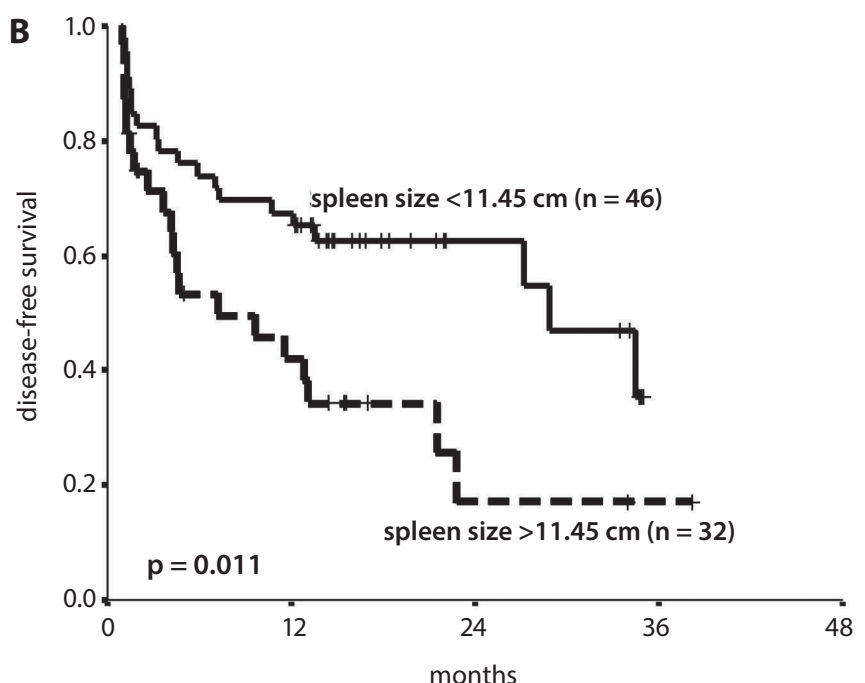

Fig. 2. Prognostic value of splenomegaly $(>11.45 \mathrm{~cm}$ ) in cirrhotic patients with hepatocellular carcinoma (HCC) following curative resection: A. overall survival; B. disease-free survival 
criteria were used for tumor histological grading. ${ }^{15}$ In addition, 5 blood-based liver function parameters, including albumin, alanine aminotransferase (ALT), aspartate aminotransferase (AST), total bilirubin, and platelet count, and 4 non-invasive liver fibrosis markers, including AST to ALT ratio (AAR), age-platelet index (API), AST to platelet ratio index (APRI), and Fibrosis-4 index (FIB-4) scores, were applied to predict splenomegaly. ${ }^{16-19}$ The acquisition of clinicopathologic and follow-up data was approved by the institutional Ethics Committee of Peking Union Medical College Hospital (Beijing, China).

\section{Determination of spleen size, blood-derived liver function parameters and non-invasive fibrosis markers}

Spleen size was routinely measured using ultrasonography (USG) and defined as the greatest dimension of the spleen. Serum liver function parameters, albumin, ALT, AST, and total bilirubin were detected preoperatively using
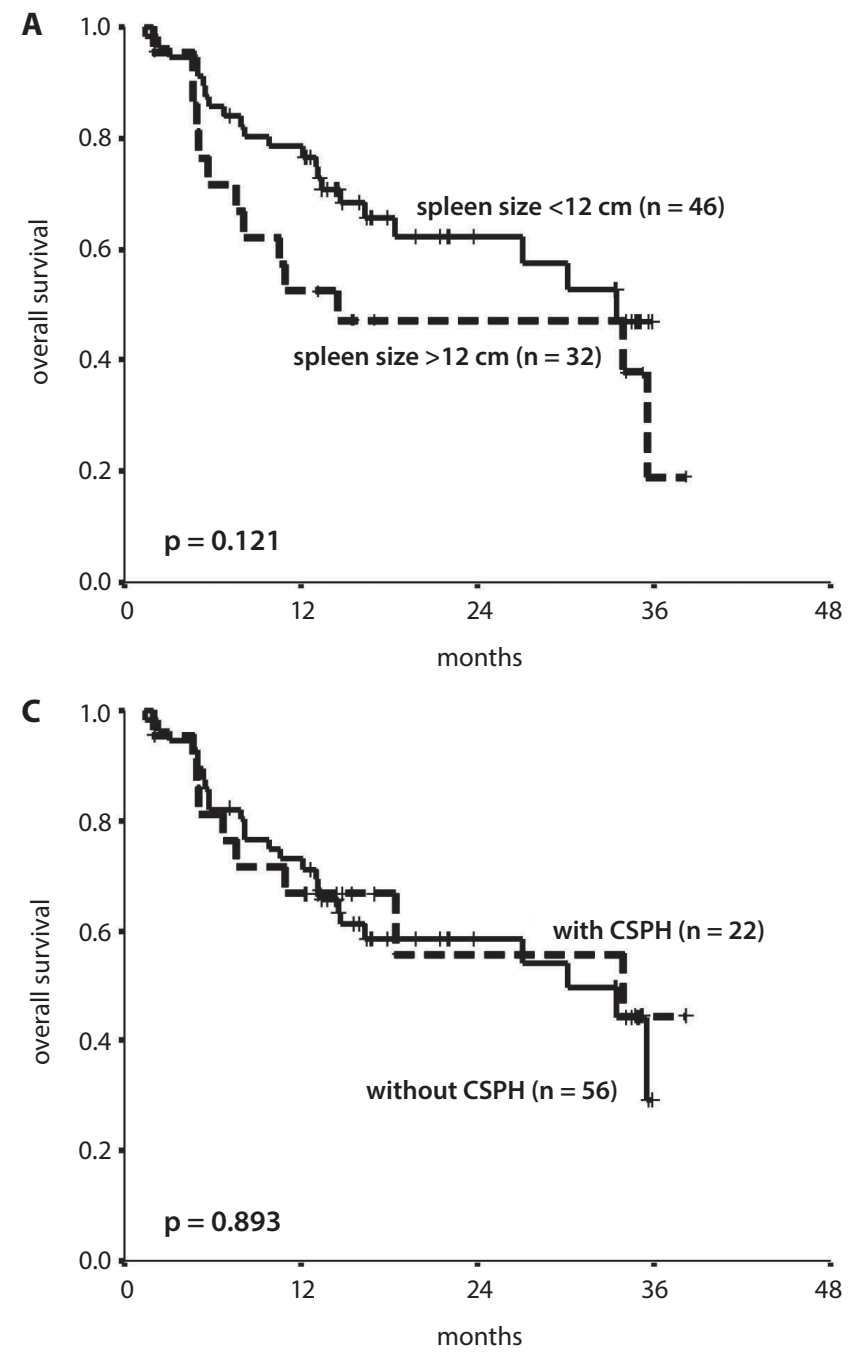

the Beckman Coulter AU5800 chemistry analyzer (Beckman Coulter Inc., Brea, USA), while the preoperative platelet number was counted using the Sysmex XE-5000 automated hematology analyzer (Sysmex Corporation, Kobe, Japan). On the basis of those results, the abovementioned non-invasive fibrosis markers were calculated according to their respective formulae. ${ }^{16-19}$

\section{Follow-up}

All the patients underwent follow-up, mainly by serum AFP level and imaging examinations, including USG, angiography, computed tomography (CT), and magnetic resonance imaging (MRI). The follow-up period ranged from 1.4 to 38.2 months (median: 14.5 months).

\section{Statistical analyses}

The cut-off value of spleen size was determined with the receiver operating characteristic (ROC) curve for survival
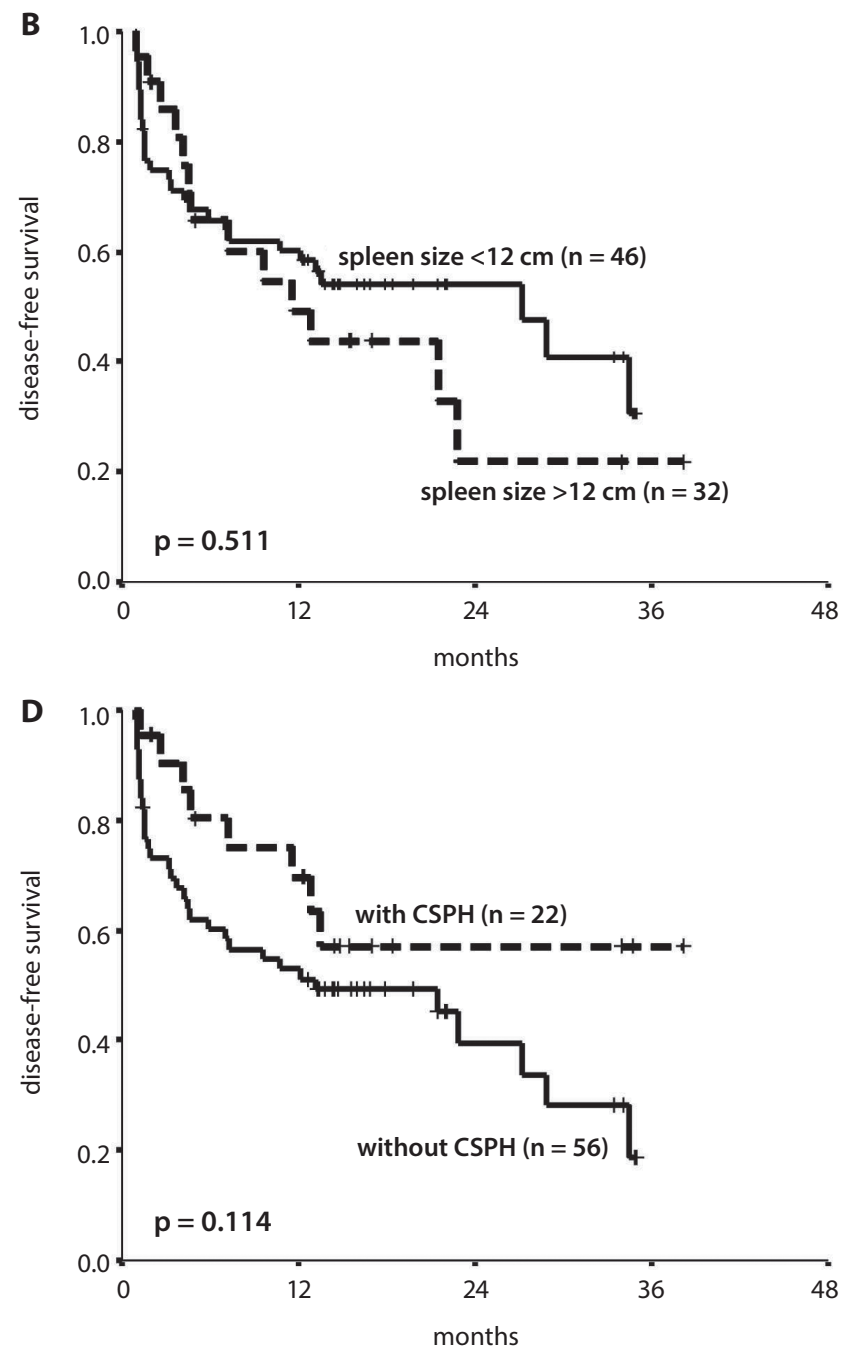

Fig. 3. Prognostic value of splenomegaly $(>12 \mathrm{~cm}$ ) and clinically significant portal hypertension (CSPH) in cirrhotic patients with hepatocellular carcinoma (HCC) following curative resection: A. splenomegaly $(>12 \mathrm{~cm})$ for overall survival; B. splenomegaly $(>12 \mathrm{~cm})$ for disease-free survival; $\mathrm{C}$. CSPH for overall survival; D. CSPH for disease-free survival 
Table 1. Univariate and multivariate analyses for the overall survival of cirrhotic patients with HCC following curative resection

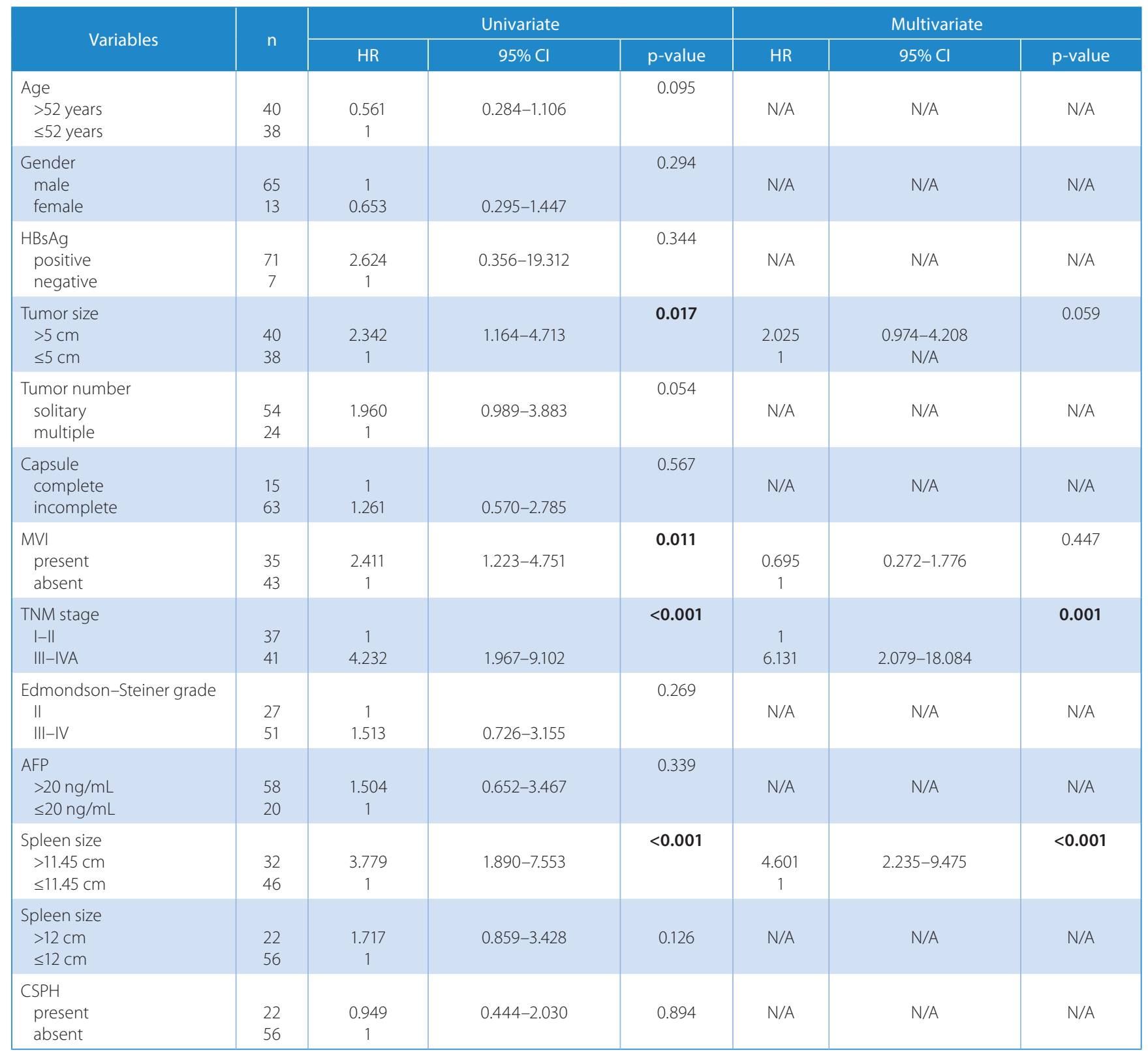

HCC - hepatocellular carcinoma; HR - hazard ratio; Cl - confidence interval; HbsAg - hepatitis B surface antigen; MVI - microvascular invasion; TNM - tumor-node-metastasis; AFP - a-fetoprotein; CSPH - clinically significant portal hypertension; values in bold are statistically significant.

and Youden's index (YI). Suvival curves of patients in the small and large spleen groups were generated using the Kaplan-Meier method and compared using the log-rank test. Univariate and multivariate Cox regression analyses (proportional hazard model) were used to identify prognostic factors. The values of the blood-derived liver function parameters and non-invasive fibrosis markers in the 2 groups were compared using the Mann-Whitney $\mathrm{U}$ test. The predictive accuracies of the variables for splenomegaly, evaluated with the area under the curve (AUC), were shown using ROC curves. SPSS statistical software package v. 11.5 (SPSS Inc., Chicago, USA) was used for all the analyses. Statistical significance was defined as $\mathrm{p}<0.05$.

\section{Results}

\section{Patient characteristics}

The study group comprised 65 male (83.3\%) and $13 \mathrm{fe-}$ male patients $(16.7 \%)$. The patients' median age was 52 years (range: $31-74$ years). There were 71 patients (91.0\%) with positive hepatitis B surface antigen (HBsAg). Clinically significant portal hypertension was present in 22 patients $(28.2 \%)$ and absent in $56(71.8 \%)$. The median tumor size was $5.2 \mathrm{~cm}$ (range: $0.3-17 \mathrm{~cm}$ ). Solitary tumors were found in 54 patients $(69.2 \%)$, whereas 15 patients (19.2\%) carried tumors with complete capsules. Microvascular invasion developed in 35 patients (44.9\%). 
Table 2. Univariate and multivariate analyses for the disease-free survival of cirrhotic patients with HCC following curative resection

\begin{tabular}{|c|c|c|c|c|c|c|c|}
\hline \multirow{2}{*}{ Variables } & \multirow{2}{*}{$\mathrm{n}$} & \multicolumn{3}{|c|}{ Univariate } & \multicolumn{3}{|c|}{ Multivariate } \\
\hline & & HR & $95 \% \mathrm{Cl}$ & $p$-value & $\mathrm{HR}$ & $95 \% \mathrm{Cl}$ & $p$-value \\
\hline $\begin{array}{l}\text { Age } \\
>52 \text { years } \\
\leq 52 \text { years }\end{array}$ & $\begin{array}{l}40 \\
38\end{array}$ & $\begin{array}{c}0.463 \\
1\end{array}$ & $\begin{array}{c}0.246-0.871 \\
\text { N/A }\end{array}$ & 0.017 & $\begin{array}{c}0.476 \\
1\end{array}$ & $0.247-0.919$ & 0.027 \\
\hline $\begin{array}{l}\text { Gender } \\
\text { male } \\
\text { female }\end{array}$ & $\begin{array}{l}65 \\
13\end{array}$ & $\begin{array}{c}1 \\
0.894\end{array}$ & $0.394-2.026$ & 0.788 & N/A & N/A & N/A \\
\hline $\begin{array}{l}\mathrm{HBsAg} \\
\text { positive } \\
\text { negative }\end{array}$ & $\begin{array}{c}71 \\
7\end{array}$ & $\begin{array}{c}3.622 \\
1\end{array}$ & $0.496-26.461$ & 0.205 & N/A & N/A & N/A \\
\hline $\begin{array}{l}\text { Tumor size } \\
>5 \mathrm{~cm} \\
\leq 5 \mathrm{~cm}\end{array}$ & $\begin{array}{l}40 \\
38\end{array}$ & $\begin{array}{c}2.677 \\
1\end{array}$ & $1.396-5.132$ & 0.003 & $\begin{array}{c}2.330 \\
1\end{array}$ & $1.181-4.600$ & 0.015 \\
\hline $\begin{array}{l}\text { Tumor number } \\
\text { solitary } \\
\text { multiple }\end{array}$ & $\begin{array}{l}54 \\
24\end{array}$ & $\begin{array}{c}1.581 \\
1\end{array}$ & $0.822-3.042$ & 0.170 & N/A & N/A & N/A \\
\hline $\begin{array}{l}\text { Capsule } \\
\text { complete } \\
\text { incomplete }\end{array}$ & $\begin{array}{l}15 \\
63\end{array}$ & $\begin{array}{c}1 \\
1.736\end{array}$ & $0.845-3.567$ & 0.133 & N/A & N/A & N/A \\
\hline $\begin{array}{l}\text { MVI } \\
\text { present } \\
\text { absent }\end{array}$ & $\begin{array}{l}35 \\
43\end{array}$ & $\begin{array}{c}2.184 \\
1\end{array}$ & $1.172-4.070$ & 0.014 & $\begin{array}{c}0.640 \\
1\end{array}$ & $0.253-1.616$ & 0.345 \\
\hline $\begin{array}{l}\text { TNM stage } \\
\text { I-II } \\
\text { III-IVA }\end{array}$ & $\begin{array}{l}37 \\
41\end{array}$ & $\begin{array}{c}1 \\
3.404\end{array}$ & $1.736-6.677$ & $<0.001$ & $\begin{array}{c}1 \\
3.806\end{array}$ & $1.408-10.286$ & 0.008 \\
\hline $\begin{array}{l}\text { Edmondson-Steiner grade } \\
\text { II } \\
\text { III-IV }\end{array}$ & $\begin{array}{l}27 \\
51\end{array}$ & $\begin{array}{c}1 \\
1.206\end{array}$ & $0.629-2.314$ & 0.573 & N/A & N/A & $\mathrm{N} / \mathrm{A}$ \\
\hline $\begin{array}{l}\text { AFP } \\
\qquad 20 \mathrm{ng} / \mathrm{mL} \\
\leq 20 \mathrm{ng} / \mathrm{mL}\end{array}$ & $\begin{array}{l}58 \\
20\end{array}$ & $\begin{array}{c}1.446 \\
1\end{array}$ & $0.681-3.072$ & 0.337 & N/A & N/A & N/A \\
\hline $\begin{array}{l}\text { Spleen size } \\
>11.45 \mathrm{~cm} \\
\leq 11.45 \mathrm{~cm}\end{array}$ & $\begin{array}{l}32 \\
46\end{array}$ & $\begin{array}{c}2.181 \\
1\end{array}$ & $1.176-4.047$ & 0.013 & $\begin{array}{c}2.280 \\
1\end{array}$ & $1.197-4.345$ & 0.012 \\
\hline $\begin{array}{l}\text { Spleen size } \\
>12 \mathrm{~cm} \\
\leq 12 \mathrm{~cm}\end{array}$ & $\begin{array}{l}22 \\
56\end{array}$ & $\begin{array}{c}1.247 \\
1\end{array}$ & $0.644-2.412$ & 0.513 & N/A & N/A & N/A \\
\hline $\begin{array}{l}\text { CSPH } \\
\text { present } \\
\text { absent }\end{array}$ & $\begin{array}{l}22 \\
56\end{array}$ & $\begin{array}{c}0.542 \\
1\end{array}$ & $0.250-1.175$ & 0.121 & N/A & N/A & N/A \\
\hline
\end{tabular}

HCC - hepatocellular carcinoma; HR - hazard ratio; Cl - confidence interval; HbsAg - hepatitis B surface antigen; MVI - microvascular invasion; TNM - tumor-node-metastasis; AFP - a-fetoprotein; CSPH - clinically significant portal hypertension; values in bold are statistically significant.

Serum AFP level was higher than $20 \mathrm{ng} / \mathrm{mL}$ in 58 patients (74.4\%). Stage I, II, III, and $\mathrm{IV}_{\mathrm{A}}$ tumors were found in 13 , 24,35 , and 6 patients $(16.7 \%, 30.7 \%, 44.9 \%$, and $7.7 \%)$, respectively, while 27,48 and 3 patients (34.6\%, 61.5\% and $3.9 \%$ ) had Edmondson-Steiner grade II, III and IV tumors, respectively.

\section{The prognostic value of splenomegaly in cirrhotic patients with hepatocellular carcinoma following curative resection}

Using the ROC curve (Fig. 1), the point $(11.45 \mathrm{~cm})$ with the highest YI of spleen size for survival was defined as the cut-off value. The patients with large spleen size had significantly poorer overall and disease-free survival than those with small spleen size $(\mathrm{p}<0.001$ and $\mathrm{p}=0.011$, Fig. 2). However, when $12 \mathrm{~cm}$ (which is included in the standard surrogate criteria) was used as the cut-off value, spleen size was not prognostic ( $p=0.121$ and 0.511, Fig. 3A,B). Furthermore, CSPH was not a significant marker for either overall or disease-free survival ( $\mathrm{p}=0.893$ and 0.114 , Fig. 3C,D). The univariate Cox regression model identified tumor size, MVI, TNM stage, spleen size, and age as predictors for overall and/or disease-free survival (all p $<0.05$, Table 1,2). In the multivariate analyses, spleen size was an independent predictor for both overall and disease-free survival (hazard ratio $(\mathrm{HR})=4.601,95 \%$ confidence interval 

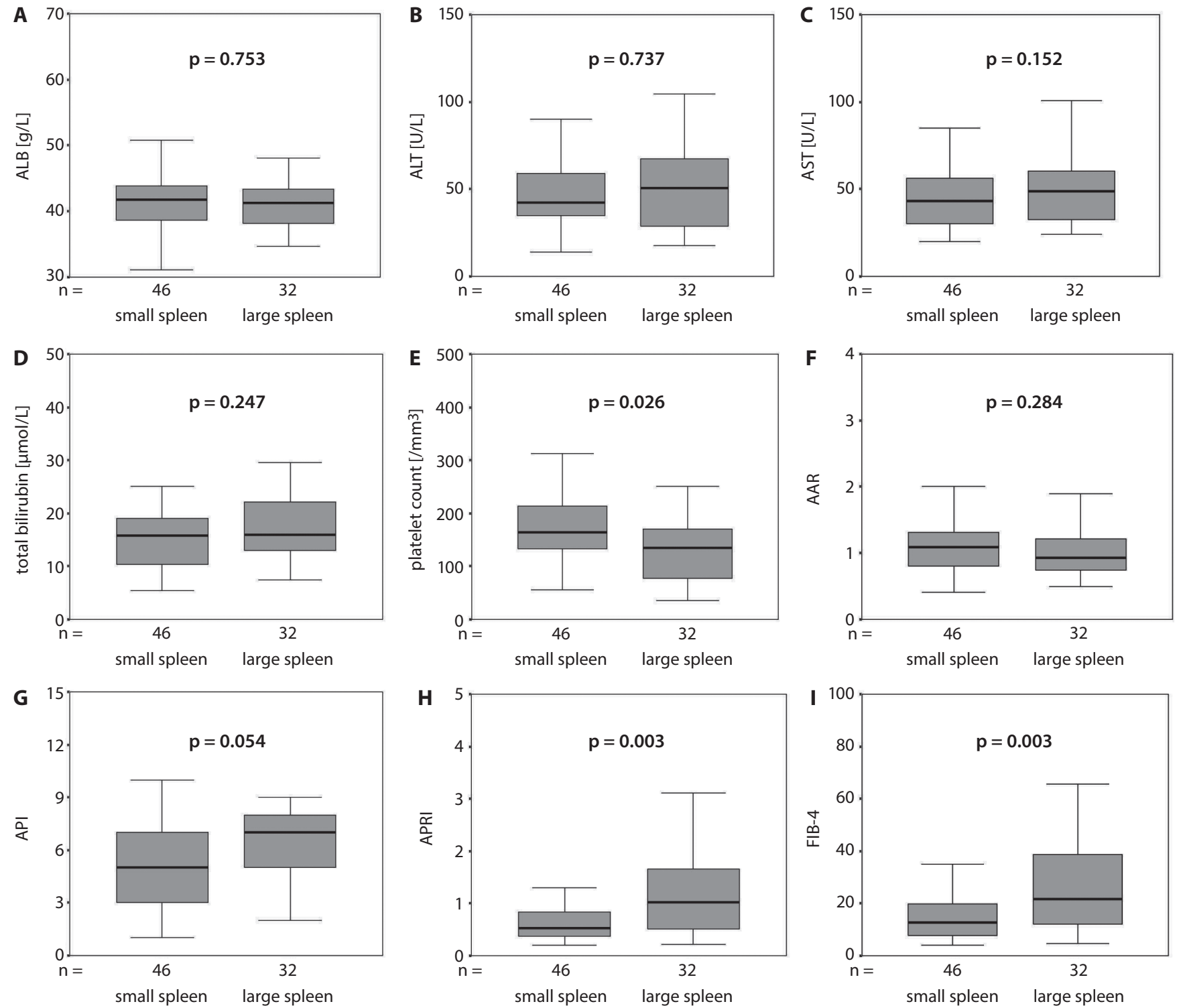

Fig. 4. Comparison of liver function parameters and non-invasive fibrosis markers between small ( $\leq 11.45 \mathrm{~cm})$ and large (>11.45 cm) spleen groups: A. albumin (ALB); B. alanine aminotransferase (ALT); C. aspartate aminotransferase (AST); D. total bilirubin; E. platelet count; F. AST to ALT ratio (AAR); G. age-platelet index (API); H. AST to platelet ratio index (APRI); I. Fibrosis-4 index (FIB-4)

$(95 \% \mathrm{CI})=2.235-9.475, \mathrm{p}<0.001$ for overall survival; $\mathrm{HR}=2.280,95 \% \mathrm{CI}=1.197-4.345, \mathrm{p}=0.012$ for diseasefree survival; Tables 1 and 2), along with TNM stage, tumor size and age (all $\mathrm{p}<0.05$, Table 1,2$)$.

\section{Predictive efficiency of blood-derived liver function parameters and non-invasive fibrosis markers for splenomegaly}

It was revealed that there were significant differences in platelet count, APRI and FIB- 4 between the small and large spleen groups $(\mathrm{p}=0.026,0.003$ and 0.003 , respectively, Fig. 4E,H,I), whereas the differences for albumin, ALT, AST, total bilirubin, AAR, and API were not statistically significant (Fig. 4A-D,F,G). The ROC curves demonstrated that platelet count, APRI and FIB-4 had high accuracy in predicting splenomegaly $(\mathrm{AUC}=0.649$,
0.702 and 0.698 , respectively, $\mathrm{p}<0.05$, Fig. 5E,H,I), while the other parameters were not of significance $(\mathrm{p}>0.05$, Fig. 5A-D,F,G).

\section{Discussion}

Splenomegaly has long been well-known as an obvious sign of portal hypertension that is frequently induced by liver cirrhosis in clinical practice. It is listed as 1 of 3 factors in the standard surrogate criteria of $\mathrm{CSPH} .{ }^{10}$ It has previously been suggested that splenomegaly has a negative impact on the overall survival of patients with HCC treated with radiofrequency ablation; however, its prognostic significance in curatively resected $\mathrm{HCC}$ has not been elucidated. ${ }^{20}$ The current study showed that splenomegaly, using the cut-off value derived from the ROC curve $(11.45 \mathrm{~cm})$, 

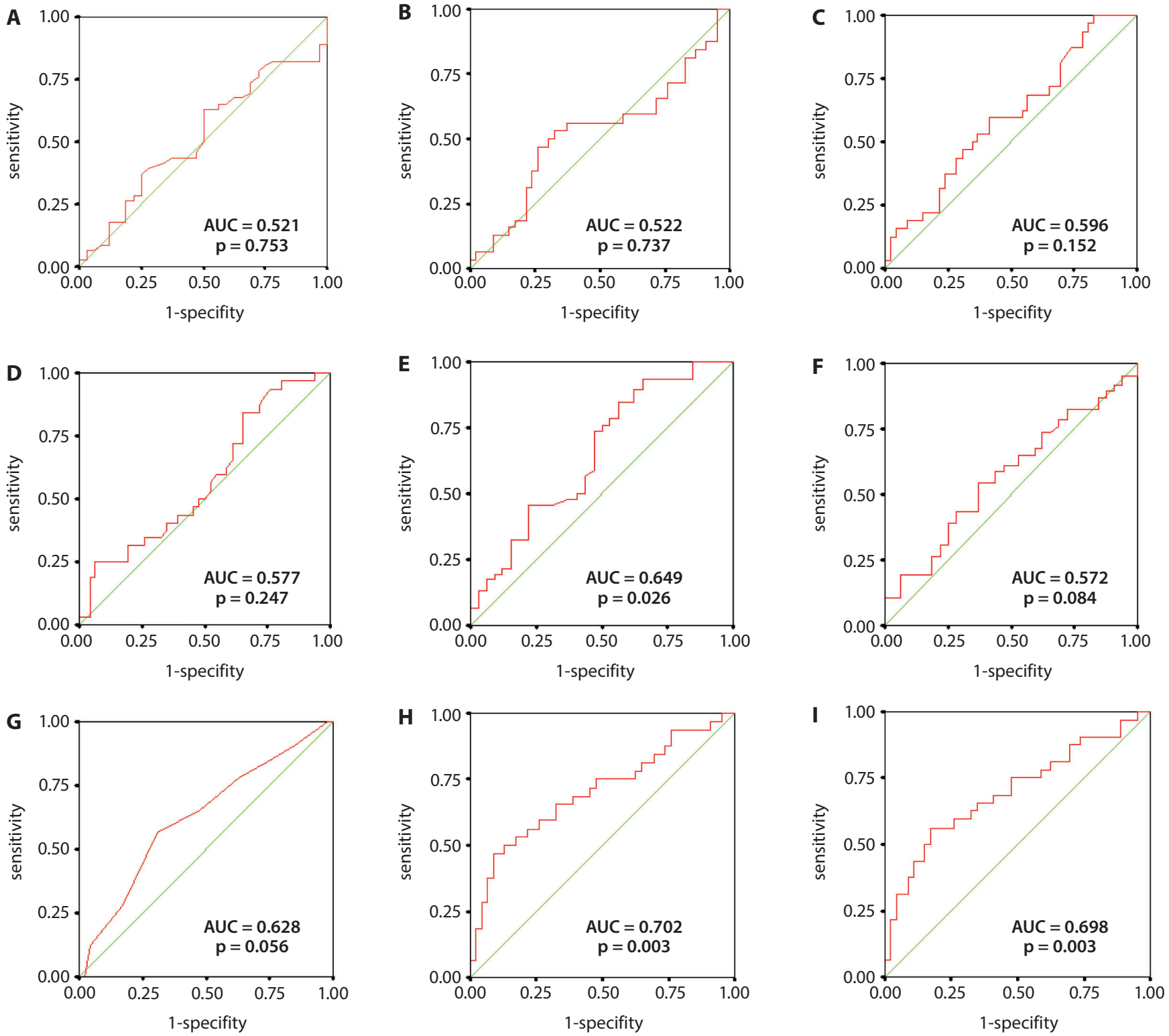

Fig. 5. Predictive accuracies of liver function parameters and non-invasive fibrosis markers for splenomegaly (>11.45 cm): A. albumin (ALB); B. alanine aminotransferase (ALT); C. aspartate aminotransferase (AST); D. total bilirubin; E. platelet count; F. AST to ALT ratio (AAR); G. age-platelet index (API); H. AST to platelet ratio index (APRI); I. Fibrosis-4 index (FIB-4); AUC - area under curve

was identified as a powerful predictor for a poor prognosis using 3 statistical tools (the log-rank test, as well as univariate and multivariate Cox regression models). These results might indicate that splenomegaly can be a promising and reliable independent prognostic marker of $\mathrm{HCC}$ before surgery. It should be emphasized that this variable was not only associated with overall survival, as previously found, but also with disease-free survival in HCC patients who had undergone curative resection. ${ }^{20}$ Thus, this study is the first to indicate the role of splenomegaly in postsurgical recurrence of HCC. However, splenomegaly $(>12 \mathrm{~cm})$ was not significant to either overall or disease-free survival, indicating that this cut-off point might not be suitable for outcome prediction after resection for HCC, although it is included in the standard surrogate criteria for CSPH. ${ }^{10}$
We also found that CSPH, determined using the standard surrogate criteria, was also not prognostic in curatively resected $\mathrm{HCC}$, which supports some previous investigations, including 1 from China. ${ }^{1-13}$ It has already been demonstrated that liver cirrhosis/fibrosis-related peritumoral ductular reaction contributes to aggressive tumor behaviors, recurrence and poor survival, and to molecular alterations, such as nuclear translocation of $\beta$-catenin and upregulated $p S m a d 2$ expression, in HCC. ${ }^{21,22}$ Therefore, the inconsistent results concerning the prognostic role of CSPH suggest an urgent need for further validation of the standard surrogate criteria, because measurement of the hepatic venous pressure gradient (HVPG), regarded as the gold diagnostic criterion for $\mathrm{CSPH}$, is not routinely used in clinical practice due to its invasiveness. On the 
other hand, in comparison to total splenic volume calculations based on 3D reconstructions of spleen CT data, measurements of spleen size using USG might be simpler, more convenient and easier to apply in clinical practice. ${ }^{20}$ Recently, it was shown that HVPG could be precisely predicted by the CT-based liver/spleen volume ratio. ${ }^{23}$ In the future, determining whether USG could be used to predict HVPG might be of interest.

In addition, it was believed that the accuracy of USGbased spleen assessment could be significantly influenced by the examiner's experience, especially due to irregular contours of the spleen and overlapping with bone, kidney or bowel gas. ${ }^{24}$ For inexperienced examiners or under difficult conditions, variables to aid in assessments might be of particular significance. Thus far, many non-invasive markers for predicting liver fibrosis and cirrhosis, such as AAR, API, APRI, and FIB-4, have been developed, based mainly on platelet number, liver biochemical parameters and age, and have been shown to have strong predictive power. ${ }^{16-19}$ However, a counterexample was also shown. ${ }^{25}$ Previously, API, APRI, FIB-4, and the platelet number were found to be effectively predictive for splenomegaly in HCC patients who underwent radiofrequency ablation. ${ }^{20}$ The present study is the first validation of the efficiency of those parameters and routine liver function tests for splenomegaly in curatively resected HCC. Like Wu et al., we suggested that platelet number, APRI and FIB-4, unlike AAR, are significant variables, while albumin, ALT, AST, and total bilirubin are not of significance. ${ }^{20}$ However, the predictive power of API in resected HCC deserves to be further confirmed, because its p-values were very close to the statistical limit in both direct comparison and the ROC curve.

\section{Conclusions}

Our data indicated that splenomegaly $(>11.45 \mathrm{~cm})$, which can be predicted by some non-invasive variables, serves as a powerful determinant for postresectional prognoses of cirrhotic patients with HCC. However, the present work might be limited by its retrospective design and relatively small sample size. The unsatisfactory AUCs of significant parameters (around 0.7) might be associated with the latter. In the future, further large-scale prospective studies that address these issues are expected.

\section{References}

1. El-Serag HB. Hepatocellular carcinoma. N Engl J Med. 2011;365(12): 1118-1127.

2. Chen W, Zheng R, Baade PD, et al. Cancer statistics in China, 2015. CA Cancer J Clin. 2016;66(2):115-132.

3. Kluger MD, Salceda JA, Laurent A, et al. Liver resection for hepatocellular carcinoma in 313 Western patients: Tumor biology and underlying liver rather than tumor size drive prognosis. $J$ Hepatol. 2015;62(5):1131-1140.
4. Chen HW, Shan L, Wan-Yee L, et al. Prognostic impact of hepatic resection for hepatocellular carcinoma. The role of the surgeon in achieving R0 resection: A retrospective cohort study. Int J Surg. 2015; 13:297-301.

5. Xu XS, Chen W, Miao RC, et al. Survival analysis of hepatocellular carcinoma: A comparison between young patients and aged patients. Chin Med J (Engl). 2015;128(13):1793-1800.

6. Goh BK, Teo JY, Chan CY, et al. Importance of tumor size as a prognostic factor after partial liver resection for solitary hepatocellular carcinoma: Implications on the current AJCC staging system. J Surg Oncol. 2016;113(1):89-93.

7. Liu W, Wang K, Bao Q, Sun Y, Xing BC. Hepatic resection provided longterm survival for patients with intermediate and advanced-stage resectable hepatocellular carcinoma. World J Surg Oncol. 2016;14:62.

8. Ishizawa T, Hasegawa K, Aoki T, et al. Neither multiple tumors nor portal hypertension are surgical contraindications for hepatocellular carcinoma. Gastroenterology. 2008;134(7):1908-1916.

9. Tang YH, Zhu WJ, Wen TF. Influence of clinically significant portal hypertension on hepatectomy for hepatocellular carcinoma: A metaanalysis. Asian Pac J Cancer Prev. 2014;15(4):1649-1654.

10. Berzigotti A, Reig M, Abraldes JG, Bosch J, Bruix J. Portal hypertension and the outcome of surgery for hepatocellular carcinoma in compensated cirrhosis: A systematic review and meta-analysis. Hepatology. 2015;61(2):526-536.

11. Cucchetti A, Ercolani G, Vivarelli M, et al. Is portal hypertension a contraindication to hepatic resection? Ann Surg. 2009;250(6):922-928.

12. Santambrogio R, Kluger MD, Costa $M$, et al. Hepatic resection for hepatocellular carcinoma in patients with Child-Pugh's A cirrhosis: Is clinical evidence of portal hypertension a contraindication? HPB (Oxford). 2013;15(1):78-84.

13. He $W$, Zeng $Q$, Zheng $Y$, et al. The role of clinically significant portal hypertension in hepatic resection for hepatocellular carcinoma patients: A propensity score-matching analysis. BMC Cancer. 2015; 15:263.

14. Cheng $\mathrm{CH}$, Lee $\mathrm{CF}$, Wu TH, et al. Evaluation of the new AJCC staging system for resectable hepatocellular carcinoma. World J Surg Oncol. 2011;9:114.

15. Edmondson HA, Steiner PE. Primary carcinoma of the liver: A study of 100 cases among 48,900 necropsies. Cancer. 1954;7(3):462-503.

16. Su CW, Chan CC, Hung HH, et al. Predictive value of aspartate aminotransferase to alanine aminotransferase ratio for hepatic fibrosis and clinical adverse outcomes in patients with primary biliary cirrhosis. J Clin Gastroenterol. 2009;43(9):876-883.

17. Poynard T, Bedossa P. Age and platelet count: A simple index for predicting the presence of histological lesions in patients with antibodies to hepatitis C virus. METAVIR and CLINIVIR Cooperative Study Groups. J Viral Hepat. 1997;4(3):199-208.

18. Wai CT, Greenson JK, Fontana RJ, et al. A simple noninvasive index can predict both significant fibrosis and cirrhosis in patients with chronic hepatitis C. Hepatology. 2003;38(2):518-526.

19. Vallet-Pichard A, Mallet V, Nalpas B, et al. FIB-4, an inexpensive and accurate marker of fibrosis in $\mathrm{HCV}$ infection: Comparison with liver biopsy and fibrotest. Hepatology. 2007;46(1):32-36.

20. Wu WC, Chiou YY, Hung HH, et al. Prognostic significance of computed tomography scan-derived splenic volume in hepatocellular carcinoma treated with radiofrequency ablation. J Clin Gastroenterol. 2012;46(9):789-795.

21. Xu M, Xie F, Qian G, et al. Peritumoral ductular reaction: A poor postoperative prognostic factor for hepatocellular carcinoma. BMC Cancer. 2014;14:65.

22. Cai $X$, Li F, Zhang $Q$, et al. Peritumoral ductular reaction is related to nuclear translocation of $\beta$-catenin in hepatocellular carcinoma. Biomed Pharmacother. 2015;76:11-16.

23. Iranmanesh $\mathrm{P}$, Vazquez $\mathrm{O}$, Terraz $\mathrm{S}$, et al. Accurate computed tomography-based portal pressure assessment in patients with hepatocellular carcinoma. J Hepatol. 2014;60(5):969-974.

24. Ishibashi H, Higuchi N, Shimamura R, Hirata Y, Kudo J, Niho Y. Sonographic assessment and grading of spleen size. J Clin Ultrasound. 1991;19(1):21-25.

25. Eminler AT, Ayyildiz T, Irak K, et al. AST/ALT ratio is not useful in predicting the degree of fibrosis in chronic viral hepatitis patients. Eur J Gastroenterol Hepatol. 2015;27(12):1361-1366. 